\title{
Therapeutic Plasma Exchange in Thyroid Storm Refractory to Conventional Treatment
}

\author{
Harold Henrison C. Chiu, RCh, MD, ${ }^{1}$ Jim Paulo D. Sarsagat, MD, ${ }^{1}$ Hydelene B. Dominguez, MD, ${ }^{1}$ \\ Ramon B. Larrazabal Jr., MD, ${ }^{2}$ Josephine Anne C. Lucero, MD, ${ }^{3}$ \\ Angelique Bea C. Uy, MD' ${ }^{1}$ and Elizabeth Paz-Pacheco, MD ${ }^{1}$ \\ ${ }^{1}$ Division of Endocrinology, Diabetes and Metabolism, Department of Medicine, Philippine General Hospital, University of the Philippines Manila \\ ${ }^{2}$ Department of Medicine, Philippine General Hospital, University of the Philippines Manila \\ ${ }^{3}$ Division of Hematology, Department of Medicine, Philippine General Hospital, University of the Philippines Manila
}

\begin{abstract}
Thyroid storm is a life-threatening condition with mortality rates reaching up to 20 to $30 \%$. First-line treatment includes inhibition of thyroid hormone synthesis, prevention of release of preformed hormones, blocking of peripheral FT4 to FT3 conversion, enhancing hormone clearance, and definitive radioactive iodine ablation. However, in the presence of life-threatening adverse effects (e.g., agranulocytosis) and contraindications (e.g., fulminant hepatic failure), therapeutic plasma exchange (TPE) can be used to rapidly remove circulating thyroid hormones, antibodies, and cytokines in plasma; this is recommended by the American Society of Apheresis (ASFA) and the American Thyroid Association (ATA) as second-line treatment for thyroid storm. Here, we report a 49-year-old female with Graves' disease admitted in our emergency room for a 6-week history of fever, weight loss, jaundice, exertional dyspnea, palpitations, and diarrhea. Her initial thyroid hormone levels were: FT4 64.35 (NV 9.01-19.05 pmol/L), FT3 23.91 (NV: $2.89-4.88 \mathrm{pmol} / \mathrm{L}$ ), and TSH 0.00000 (NV: 0.35-4.94 mlU/L) and we managed her as a case of thyroid storm (Burch-Wartofsky score 70) by initiating high dose propylthiouracil. However, her sensorium deteriorated and serum bilirubin continued to rise from 307.2 on admission to $561.6 \mathrm{umol} / \mathrm{L}$ on the $5^{\text {th }}$ hospital day (NV: $3-22 \mathrm{umol} / \mathrm{L}$ ). TPE was performed after consultation with the Division of Hematology. Over the treatment course, her thyroid hormones normalized: FT4 $13.18 \mathrm{pmol} / \mathrm{L}, \mathrm{FT} 32.30 \mathrm{pmol} / \mathrm{L}$. However, despite TPE, her symptoms worsened and she became comatose, had hypotension despite vasopressors and developed new-onset atrial fibrillation. She expired on her $7^{\text {th }}$ hospital day from multiorgan failure. TPE is effective in decreasing circulating thyroid hormone levels. However, it had no effect on clinically important outcomes as our patient still deteriorated and eventually succumbed. We still wrote and submitted this case report since if only successful cases were reported, the true effectiveness rate of TPE could not be determined.
\end{abstract}

Key Words: thyroid storm; thyrotoxicosis; therapeutic plasma exchange

\section{INTRODUCTION}

Thyroid storm is a preventable, severe, and life-threatening progression of thyrotoxicosis, characterized by dysfunction of the thermoregulatory, central nervous, gastrointestinal, hepatic, and cardiovascular systems. The mortality rate is high, reaching up to about 20 to $30 \% .{ }^{1}$ Medical management has mainly focused on the use of (1) medications that inhibit

Poster presented in the $20^{\text {th }}$ ASEAN Federation of Endocrine Societies Congress on November 21-23, 2019, at the Philippine International Convention Center, Manila, Philippines.

Corresponding author: Harold Henrison C. Chiu, RCh, MD Division of Endocrinology, Diabetes and Metabolism Department of Medicine Philippine General Hospital

University of the Philippines Manila,

Taft Avenue, Ermita, Manila 1000, Philippines

Email: harold.c.chiu@gmail.com, hcchiu@up.edu.ph the synthesis (i.e., thionamides), release (i.e., iodide), and peripheral conversion (i.e., propylthiouracil and high dose beta blockers) of thyroid hormones, and (2) supportive measures that aim to address the excessive sympathetic adrenergic milieu. However, the efficacy of antithyroid medications is sometimes limited because of rare but serious side effects or from failure to control disease progression. Furthermore, the long half-life of thyroxine contributes to its high effective circulating levels and does not permit a 
rapid decrease in levels through inhibition of synthesis and peripheral conversion alone. ${ }^{2}$

Therapeutic plasma exchange (TPE) and plasmapheresis (PP) are effective alternative therapies for the treatment of thyrotoxicosis and thyroid storm as both procedures can effectively reduce circulating thyroid hormone levels within 3 days. ${ }^{3}$ As early as the late 1960s, plasma exchange has been used in thyroid storm patients who failed to respond to conventional medical therapy, removing thyroid hormones that are bound to plasma proteins. Albeit the transient effect of PP/TPE on thyrotoxicosis, lasting only an approximate 24 to 48 hours, it can be used as temporizing and bridging intervention to rapidly normalize thyroid hormone levels prior to definitive therapy. The overall incidence of adverse effects remains low at approximately five percent including hypotension, hemolysis, anaphylactic or allergic reactions, coagulopathy, vascular injury, and infection.

\section{MATERIALS AND METHODS}

The study was carried out in accordance with the principles outlined in the 2008 Declaration of Helsinki. Informed consent was obtained from the patient prior to the beginning of the study.

\section{CASE PRESENTATION}

A forty-nine-year-old woman was admitted in our emergency room for a six-week history of progressive dyspnea described as an increase in her baseline exertional dyspnea, from dyspnea on 2 flights of stairs, to less than ordinary activity on admission. She also experienced occasional chest heaviness, palpitations, undocumented episodes of fever, night sweats, and progressive weight loss. She had been diagnosed to have Graves' disease for 9 years and was previously on propylthiouracil, however, she stopped taking her medications due to financial constraints. During the interim, her symptoms progressed to paroxysmal nocturnal dyspnea, jaundice, and bipedal edema.

Important physical examination findings included: cachexia, exophthalmos, icteric sclerae, a well-defined $4.0 \mathrm{x}$ $3.0 \times 3.0 \mathrm{~cm}$ anterior neck mass that moved with deglutition, bibasal crackles on chest auscultation, decreased breath sounds and fremiti, left base, tachycardia at 150 beats per minute, grade 1 bipedal edema, and tremors. Initial diagnostic examinations revealed the following: decreased TSH with increased FT3 and FT4, hypokalemia, and deranged PTINR, elevated total bilirubin (16x), AST (4x), and ALT (3x), and ECG findings of sinus tachycardia, normal axis, left ventricular hypertrophy.

She was managed as a case of Graves' disease in storm, with a Burch and Wartofsky score of 70 and was given oral propranolol $40 \mathrm{mg}$, propylthiouracil $600 \mathrm{mg}$, and intravenous hydrocortisone $100 \mathrm{mg}$. Her medications were continued as follows: propylthiouracil at $200 \mathrm{mg}$ every 4 hours, intra- venous hydrocortisone $100 \mathrm{mg}$ intravenously every 8 hours, propranolol $40 \mathrm{mg}$ every 6 hours, oral potassium chloride, and vitamin $\mathrm{K}$.

However, despite the timely intervention, her condition worsened, with deteriorating sensorium requiring intubation for airway protection and with progressive liver failure. From admission until the $5^{\text {th }}$ hospital day, the total bilirubin rose from $307.2(13.3 \mathrm{x})$ to $561.6(25.7 \mathrm{x})$ umol/L (NV: 3 $22 \mathrm{umol} / \mathrm{L}$ ); ALT rose from 324 (9x) U/L to 1606 (44.6x) U/L (NV: 14 - $36 \mathrm{U} / \mathrm{L})$; AST rose from 866 (24.7x) U/L to 3394 (97x) U/L (NV: < 35 U/L), and INR increased from 2.1 to 9.69 . The worsening liver function tests precluded the further use of high doses of antithyroid medications propylthiouracil and methimazole. She was also deemed a poor surgical candidate with a high risk of developing major adverse cardiovascular events during total thyroidectomy. Due to the inadequate response to initial management, therapeutic plasma exchange of one plasma volume was performed on the third hospital day using saline-albumin as the replacement fluid.

Over the treatment course, her thyroid hormones decreased: FT3 (23.91 pmol/L on admission to $2.30 \mathrm{pmol} / \mathrm{L}$, 24 hours post TPE) and FT4 (64.35 pmol/L on admission to $13.18 \mathrm{pmol} / \mathrm{L}, 24$ hours post TPE). Further plasma exchange was not attempted due to the critical hemodynamic status of the patient. She remained symptomatic and continued to deteriorate with baseline sinus rhythm progressing to atrial fibrillation refractory to both medical and electrical therapy. During her $5^{\text {th }}$ hospital day at the intensive care unit, her sensorium further deteriorated to being comatose, and she was persistently in shock despite vasopressors. She eventually expired on her $7^{\text {th }}$ hospital day from multiorgan failure precluding the determination of additional thyroid hormone levels.

\section{DISCUSSION}

Historically, diagnosis of thyroid storm is based on clinical symptoms scored using the Burch and Wartofsky scoring, with a score of 45 being indicative of storm. ${ }^{4}$ In 2012, the Japan Thyroid Association proposed a new set of diagnostic criteria that assigned two grades based on signs and symptoms. ${ }^{5}$ Along with elevated levels of T3 and T4, other parameters should include either cardiovascular symptoms (i.e., tachycardia disproportionate to the fever) or gastrointestinal symptoms (i.e., nausea, diarrhea), and neurological symptoms (i.e., agitation, delirium, psychosis, stupor, or coma). Our patient fulfilled the criteria for TS1. ${ }^{5}$ The management of thyroid storm requires a multimodality treatment approach. ${ }^{6}$ The general approach includes inhibition of thyroid hormone synthesis (i.e., propylthiouracil (preferred drug) or methimazole), prevention of release of preformed hormones (i.e., iodine or iodide salts), blocking of T4 to T3 peripheral conversion (i.e., propylthiouracil and dexamethasone), enhancing hormone clearance (i.e., 
cholestyramine), addressing the peripheral effects of the thyroid hormones (i.e., beta-blockers), supportive treatment of high fever (i.e., paracetamol, cooling blankets), and the treatment of the precipitating or underlying cause. ${ }^{6}$ Large doses of propylthiouracil (PTU) consisting of 500-1000 $\mathrm{mg}$ loading doses followed by $250 \mathrm{mg}$ every 4 hours or / and $60-80 \mathrm{mg}$ per day of methimazole in divided doses are given orally, by stomach tube, or per rectum. PTU is preferable to methimazole because it has the additional action of inhibiting peripheral conversion of T3 from T4. Controlling the cardiovascular manifestations of thyroid storm is vital. Relatively large doses (60-80 mg every 4 hours) of propranolol are usually required because of the faster metabolism of the drug, and possibly because of an increased number of cardiac beta-adrenergic receptors. ${ }^{6}$

Thyroxine (T4) has the highest concentration among iodothyronines in the plasma and is produced exclusively by the thyroid gland; triiodothyronine (T3) is primarily derived from the peripheral conversion through deiodination of T4. Two-thirds of total T4 fraction is bound to thyroxine binding globulin (TBG), around $11 \%$ to transthyretin, and $20 \%$ to albumin while T3 is $80 \%$ bound to TBG, $9 \%$ to transthyretin, and $11 \%$ to albumin. ${ }^{7}$

TPE and PP both utilize the advantage of extensive protein binding in the clearance of thyroid hormones. TPE and $\mathrm{PP}$ are extracorporeal blood purification techniques used for eliminating large molecular substances from the plasma. In both methods, the patient's plasma is separated and removed from other blood components. On one hand, PP removes the patient's plasma and does not require any form of replacement fluid. On the other hand, in TPE, plasma removed is replaced with an equivalent of replacement fluids (i.e., fresh frozen plasma and albumin). ${ }^{3}$ Plasma used as replacement fluid has the advantage of increasing the concentration of TBG to bind free thyroid hormone. However, albumin provides a larger capacity for low-affinity binding of thyroid hormones. Human albumin solutions are given during TPE to provide new binding sites for circulating free hormones which are cleared during the next TPE session and thus, this process limits the bioavailability of free thyroid hormones. Other possible mechanisms would be the removal of TBG, circulating TSH receptor stimulating antibodies, cytokines, catecholamines, and deiodinase enzymes. ${ }^{7,8}$ Unfortunately, we were not able to determine the levels of TSH receptor antibodies pre- and post-TPE.

TPE was first used for the treatment of hyperthyroidism in 1970 by Ashkar et al. on three cases of thyroid storm..$^{1,2}$ In the latest American Society for Apheresis 2019 Guidelines ${ }^{9}$, TPE has been elevated from a Category III Level IIC recommendation ${ }^{10}$ (optimum role of apheresis therapy is not established; decision making should be individualized) to a Category II Level IIC recommendation (disorders for which apheresis is accepted as second-line therapy, either as a standalone treatment or in conjunction with other modes of treatment) for patients with thyroid storm with severe symptoms and when first-line therapies discussed above fail or cannot be used due to toxicity, such as agranulocytosis or severe liver failure. In literature, which contains mostly case reports, most patients are reported to have a decrease in the hormone concentrations and symptomatic improvement. This effect was dependent on the T4 serum level, suggesting earlier TPE is more efficient. ${ }^{9,10}$ However, the effect of PP/ TPE on thyrotoxicosis is transient, lasting approximately 2448 hours. ${ }^{10} \mathrm{PP}$ or TPE can therefore be used as temporizing and bridging interventions to rapidly normalize thyroid hormone levels prior to definitive surgical therapy. The overall incidence of adverse effects is approximately 5\%, including hypotension, hemolysis, anaphylactic or allergic reactions, coagulopathy, vascular injury, and infection.

Our literature search in PubMed using the terms: thyroid storm, plasma exchange, and plasmapheresis yielded a total of 28 articles, mostly case reports with favorable outcomes after therapeutic plasma exchange and plasmapheresis; one case series reported a negative outcome after the use of PP in thyroid storm ${ }^{11}$. Unfortunately for our patient, despite the prompt initiation of TPE, she continued to deteriorate due to the overwhelming effects of thyrotoxicosis causing multiorgan failure.

\section{CONCLUSION}

In summary, thyroid storm is a preventable but critical illness, if not treated promptly, that can result in death from multiorgan failure. Plasma exchange should be considered only as an alternative stabilizing measure, especially in patients who have severe and progressive symptoms, and are unable to tolerate first-line therapy. TPE is effective in decreasing circulating thyroid hormone levels. However, in the case of our patient, it had no effect on clinically important outcomes as she continued to deteriorate and eventually succumbed. Most case reports have reported only successful outcomes with TPE. Given the paucity of literature on unsuccessful outcomes using TPE, we submitted this case report since if only successful cases were reported, the true effectiveness rate of TPE could not be determined.

\section{Statement of Authorship}

All authors participated in data collection and analysis, and approved the final version submitted.

\section{Author Disclosure}

All authors declared no conflicts of interest.

\section{Funding Source}

This paper was self-funded.

\section{REFERENCES}

1. Alfadhli E, Gianoukakis AG. Management of severe thyrotoxicosis when the gastrointestinal tract is compromised. Thyroid. 2011;21(3): $1-6$. 
2. Ashkar FS, Katims RB, Smoak WM, Gilson AJ. Thyroid storm treatment with blood exchange and plasmapheresis. JAMA. 1970; 214(7): 1275-9.

3. Carhill A, Gutierrez A, Lakhia R, Nalini R. Surviving the storm: two cases of thyroid storm successfully treated with plasmapheresis. BMJ Case Reports. 2012; doi:10.1136/bcr-2012-006696.

4. Burch HB, Wartofsky L. Life-threatening thyrotoxicosis: thyroid storm. Endocrinol Metab Clin North Am. 1993; 22: 263-77.

5. Akamizu T, Satoh T, Isozaki O, Suzuki A, Wakino S, Iburi T, et. al. Japan Thyroid Association 2012 Diagnostic criteria, clinical features, and incidence of thyroid storm based on nationwide surveys. Thyroid. 2012; 22: 661-79.

6. Ross DS, Burch HB, Cooper DS, Greenlee MC, Laurberg P, Maia AL, et. al. 2016 American Thyroid Association Guidelines for Diagnosis and Management of Hyperthyroidism and Other Causes of Thyrotoxicosis. Thyroid. 2016; 26(16): 1343-1438.

7. Garla V, Kovvuru K, Ahuja A, Palabindala V, Malhotra B, Salim SA. Severe hyperthyroidism complicated by agranulocytosis treated with therapeutic plasma exchange: case report and review of the literature. Case Reports in Endocrinology. 2018; doi: https://doi. org/10.1155/2018/4135940.
8. Jha S, Waghdhare S, Reddi R, Bhattacharya P. Thyroid storm due to inappropriate administration of a compounded thyroid hormone preparation successfully treated with plasmapheresis. Thyroid. 2012; 22(12): 1283-6.

9. Schwartz J, Padmanabhan A, Aqui N, Balogun RA, Connelly-Smith L, Delaney M, et. al.. Guidelines on the Use of Therapeutic Apheresis in Clinical Practice-Evidence-Based Approach from the Writing Committee of the American Society for Apheresis: The Seventh Special Issue. Journal of Clinical Apheresis. 2016; 31: 149-338.

10. Padmanabhan A, Connelly-Smith L, Aqui N, Balogun RA, Klingel R, Meyer E, et. al.. Guidelines on the Use of Therapeutic Apheresis in Clinical Practice - Evidence-Based Approach from the Writing Committee of the American Society for Apheresis: The Eighth Special Issue. Journal of Clinical Apheresis. 2019; 34: 171-354.

11. Ligtenberg J, Tulleken J, Zijlstra J. Plasmapheresis in thyrotoxicosis Ann Intern Med. 1999;131(1):71-72. 